\title{
Reuse of E-plate cell sensor arrays in the xCELLigence Real-Time Cell Analyzer
}

\author{
Justyna Stefanowicz-Hajduk, Anna Adamska, Rafal Bartoszewski, and J. Renata Ochocka \\ Department of Biology and Pharmaceutical Botany, Medical University of Gdansk, Gdansk, Poland
}

BioTechniques 61:117-122 (September 2016) doi 10.2144/000114450

Keywords: impedance; sensor-electrodes; regeneration; cell index; trypsinization; RTCA system

Supplementary material for this article is available at www.BioTechniques.com/article/114450.

The xCELLigence Real-Time Cell Analyzer (RTCA) is a non-invasive, impedence-based biosensor system that can measure cell viability, migration, growth, spreading, and proliferation. Changes in cell morphology and behavior are continuously monitored in real time using microelectronics located in the wells of RTCA E-plates. According to the manufacturer's recommendation, E-plates are single-use and disposable. Here, we show that E-plates can be regenerated and reused several times without significantly effecting experimental results.

Most current biological assays are based on the use of labeling reagents followed by optical detection. One alternative to these conventional assays is the xCELLigence Real-Time Cell Analyzer (RTCA) from ACEA Biosciences, a label-free system that integrates molecular and cell biology assays with microelectronics $(1,2)$. This system allows monitoring of cellular processes by measuring the impedance of sensor-electrodes located at the bottom of each well in the RTCA E-plates. Changes in cell number, viability, morphology, and cytoskeletal dynamics are reflected by changes in the signals from the electrodes. The instrument allows non-invasive, real-time monitoring of cells that is highly sensitive, accurate, and produces a large amount of information. Therefore, the RTCA has a broad range of applications in many biological assays, not only for detecting cell adhesion, proliferation, migration, and differentiation (3-5) but also for the detection of cell- and compound-mediated cytotoxicity/apoptosis and receptor-mediated signaling (6-9). The system has been successfully applied in studies of environmental toxicity (10), cellular function (11), anticancer drug candidates (12-16), and microbiology (17-19).

The E-plates used for the RTCA system are single-use; thus, the cost of the plates might limit the number of experiments a researcher can perform. Here, we describe a procedure for regeneration and reuse of RTCA E-plates, and we show that reusing a plate multiple times does not affect the reproducibility of results obtained in cytotoxicity experiments.

\section{Materials and methods}

Dulbecco's Modified Eagle's Medium (DMEM), fetal bovine serum (FBS), penicillin, streptomycin, L-glutamine, phosphate buffered saline (PBS), 0.25\% trypsin-EDTA solution, methanol, $0.1 \%$ collagen solution from calf skin, and vinblastine sulfate were purchased from Sigma-Aldrich (St. Louis, $\mathrm{MO})$. Hank's balanced salt solution (HBSS) without phenol red and wheat germ agglutinin (WGA) Alexa Fluor 350 conjugate were obtained from Invitrogen (Carlsbad, CA). Vinblastine sulfate was dissolved in methanol at a concentration of $1 \mathrm{mM}$.

\section{Cell culture}

The human cervical adenocarcinoma cell line HeLa S3 and the human breast adenocarcinoma cell line MCF-7 were obtained from the American Type Culture Collection (ATCC) (Manassas, VA). The cells were cultured in DMEM supplemented with 10\% (v/v) FBS, $100 \mu \mathrm{g} / \mathrm{mL}$ of streptomycin, 100 $\mathrm{U} / \mathrm{mL}$ of penicillin, and $2 \mathrm{mM} \mathrm{L}$-glutamine. Cells were kept at $37^{\circ} \mathrm{C}$ in a humidified $5 \%$ $\mathrm{CO}_{2}$ incubator.
Real-time cell proliferation monitoring HeLa and MCF-7 cells were seeded at densities of $2 \times 10^{4}$ and $6 \times 10^{4} \mathrm{cells} /$ well, respectively, into an E-plate 16 (ACEA Biosciences, San Diego, CA) containing $100 \mu \mathrm{L}$ medium per well and monitored on the xCELLigence Real-Time Cell Analyzer Dual Plate (RTCA DP) instrument (ACEA Biosciences). When the cells entered log phase, the vinblastine solution was added to final concentrations of 1.0-20.0 nM. The final methanol concentration in the wells did not exceed $4 \%(\mathrm{v} / \mathrm{v})$. The cells were treated with vinblastine for $24 \mathrm{~h}$ and incubated at $37^{\circ} \mathrm{C}$ in a $5 \% \mathrm{CO}_{2}$ atmosphere. To calculate the half maximal inhibitory concentration $\left({ } \mathrm{C}_{50}\right)$ values, RTCA software v. 1.2.1 was used. All of the experiments were repeated at least 10 times.

\section{Regeneration of E-plates}

The RTCA E-plates were used up to three times in these experiments. After every use of a single plate, the wells were washed two times with PBS. Cells attached to the well bottom were trypsinized by adding $100 \mu \mathrm{L}$ trypsin-EDTA solution to every well. Next, the cells were removed, and the wells were washed twice with PBS. All of the plate preparation steps were performed in a sterile culture hood. In order to avoid electrode damage, media, buffer, and trypsin were removed by aspiration with blunt plastic pipette tips. Before each re-use

\section{METHOD SUMMARY}

The E-plates in the xCELLigence Real-Time Cell Analyzer (RTCA) system can be regenerated and used several times in cytotoxicity studies without losing the reproducibility of experimental data. Here, we present washing and trypsinization steps for reusing the plates. 



Figure 1. xCELLigence Real-Time Cell Analyzer (RTCA) impedance profiles of E-plates containing HeLa cells treated with vinblastine sulfate for $24 \mathbf{h}$. Experiments were conducted with new (A,D), once-used (B,E), and twice-used (C,F) E-plates. The plates were either uncoated (A,B,C) or coated with collagen (D,E,F). The different vinblastine sulfate concentrations (1.0, 2.0, 4.0, 6.0, 8.0, 10.0, $20.0 \mathrm{nM})$ are color-coded. The red line represents the profile of the control sample [cells treated with 4\% (v/v) $\mathrm{MeOH}$ ].

of a plate, we verified the absence of cells at the bottoms of the wells by microscopy. Furthermore, at the start of every experiment, the cell index (Cl) background signal (Cl signal without cells) for all of the wells was 0 at time point $\mathrm{O}$, confirming the complete removal of cells during trypsinization.

In the second part of our study, we used E-plates coated with a $0.01 \%$ (v/v) collagen solution (final concentration). The bottoms of the wells were coated with $6-10 \mu \mathrm{g}$ collagen $/ \mathrm{cm}^{2}$. Following protein binding to the well bottom, the excess solution was removed from the wells. The plates were then dried in a culture hood and exposed to UV light for $1 \mathrm{~h}$.

\section{Lectin staining}

A fluorescent lectin stain based on a WGA Alexa Fluor 350 conjugate was used according with the manufacturer's protocol. Briefly, the WGA conjugate was prepared as a $1.0 \mathrm{mg} / \mathrm{mL}$ stock solution in PBS and then applied to plate wells at a final concentration of $10.0 \mu \mathrm{g} / \mathrm{mL}$ in HBSS buffer. Following a 10 min incubation at $37^{\circ} \mathrm{C}$, the wells were washed twice in HBSS and examined under a fluorescent microscope (filter D; excitation 355-425 nm, emission 455 nm) (Leica Microsystems, Heerbrugg, Switzerland). Lectin labeling was performed before and after every trypsinization.

\section{Statistical analysis}

All data are expressed as mean \pm standard deviation (SD). Statistical comparisons among the results obtained from the experiments were performed using two-way ANOVA. Differences among the data were considered statistically significant if $P<0.05$.

\section{Results and discussion}

To examine the effects of reusing E-plates, we analyzed RTCA impedance profiles and

Table 1. Half maximal inhibitory concentration $\left(\mathrm{IC}_{50}\right)$ values $(\mathrm{nM})$ for vinblastine sulfate in HeLa cells obtained by the xCELLigence Real-Time Cell Analyzer (RTCA) with E-plates that were used up to three times.

\begin{tabular}{|c|c|c|c|}
\hline \multirow[t]{2}{*}{ E-plates } & \multicolumn{3}{|c|}{$\mathrm{IC}_{50}[\mathrm{nM}] ; R^{2 \mathrm{a}}$} \\
\hline & First use of plates & Second use of plates & Third use of plates \\
\hline Plates without collagen & $5.06 \pm 0.35 ; R^{2}=0.93$ & $5.22 \pm 0.56 ; R^{2}=0.98$ & $5.11 \pm 0.58 ; R^{2}=0.96$ \\
\hline Plates with collagen & $5.05 \pm 0.37 ; R^{2}=0.95$ & $5.21 \pm 0.50 ; R^{2}=0.96$ & $5.16 \pm 0.39 ; R^{2}=0.96$ \\
\hline
\end{tabular}



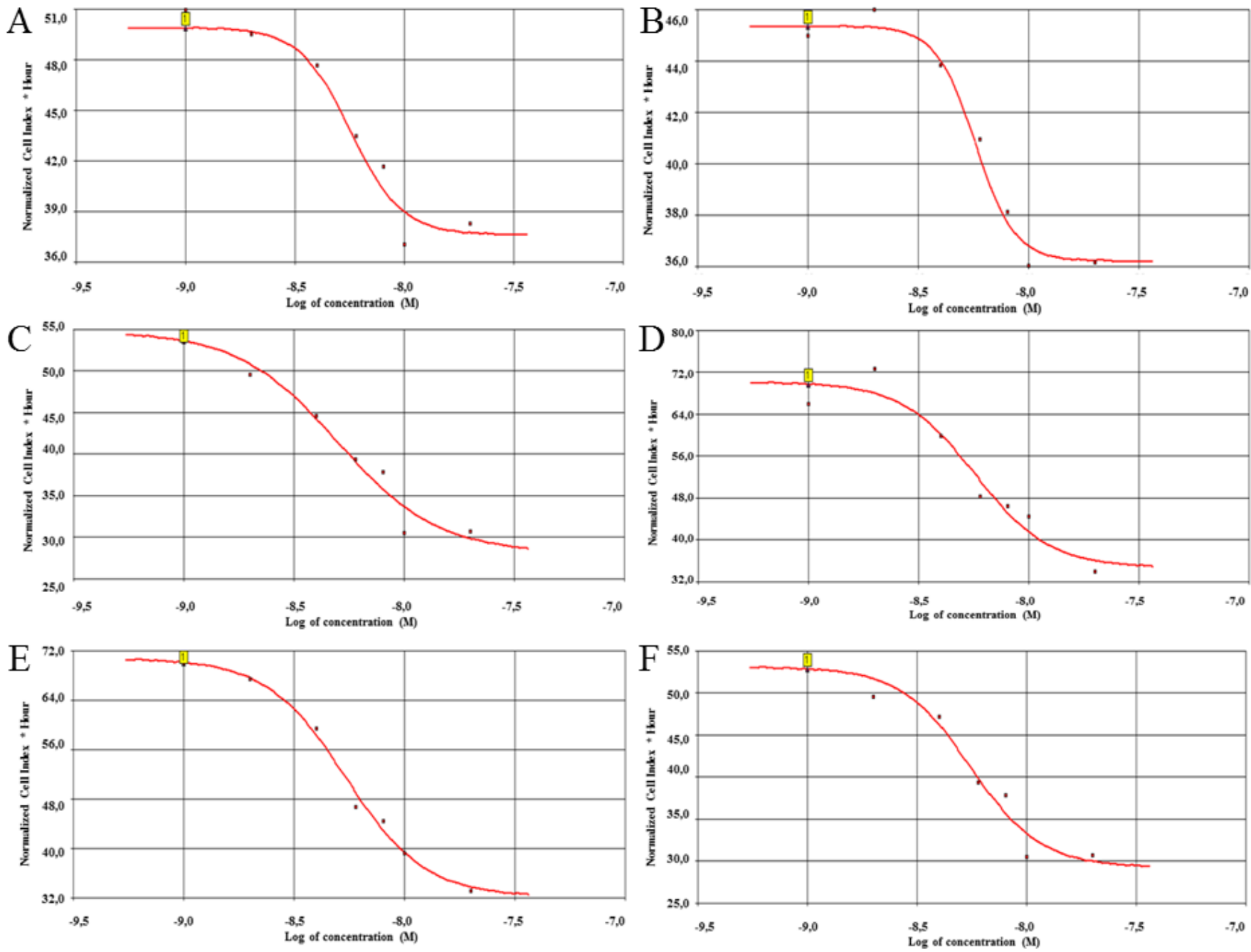

Figure 2. Half maximal inhibitory concentration $\left(\mathrm{IC}_{50}\right)$ of vinblastine sulfate after $\mathbf{2 4} \mathrm{h}$ treatment of HeLa cells from $\mathbf{6}$ different groups of E-plates. For each experiment, $I C_{5}$ values were calculated based on dose-response curves (area under the curve, dose dependent). Experiments were conducted with new (A,D), once-used $(B, E)$, and twice-used $(C, F)$ plates with $(D, E, F)$ or without $(A, B, C)$ collagen coating.

calculated the resulting $\mathrm{IC}_{50}$ values for our experiments following vinblastine sulfate treatment of HeLa and MCF-7 cells. We compared the RTCA impedance profiles obtained from six different groups of plates, which were designated as follows: A, B, Cthe new, once-used, and twice-used plates, respectively, without collagen coating; and $\mathrm{D}$, E, F-the new, once-used, and twice-used plates, respectively, with collagen coating. Furthermore, in our previous studies using RTCA, we assessed the effect of vinblastine sulfate on the HeLa cells (20).

As shown in Figure 1, the impedance profiles obtained with HeLa cells from all six of the plate groups were comparable. Furthermore, similar results were obtained using MCF-7 cells (Supplementary Figure S1).

For all impedance profiles, the sigmoidal dose-response curves (Figure 2 and Supplementary Figure S2) were obtained, and $\mathrm{IC}_{50}$ values were calculated. The calculations were based the $\mathrm{Cl}$, which is a relative and dimensionless value representing the impedance change divided by the background value and thus reflects the overall number of cells and the quality of their attachment (11). The $\mathrm{Cl}$ can change as a function of time, resulting in timedependent and dose-dependent impedance profiles during an experiment $(1,11)$.

In all experiments, the $\mathrm{IC}_{50}$ values of vinblastine sulfate ranged $5.05-5.22 \mathrm{nM}$ for HeLa cells (Table 1) and 8.24-8.39 nM for MCF-7 cells (Supplementary Table S1). Statistical analyses did not show significant differences among the $\mathrm{IC}_{50}$ values of the six different plate groups. Hence, neither trypsinization of the cells nor collagen coating influenced the cytotoxicity results.

We used a fluorescent lectin stain that selectively binds to $\mathrm{N}$-acetylglucosamine and $\mathrm{N}$-acetylneuraminic acid residues to examine the efficiency of HeLa cell (Figure 3) and MCF-7 cell (Supplementary Figure S3) trypsinization, as well as assess if residual extracellular matrix was still present on the plate surface. The surfaces of the six different groups of plates were examined before and after each reuse. As shown in Figure 3 and Supplementary Figure S3, after every trypsinization procedure, the surfaces of all tested plates were clean and devoid of extracellular debris.

The xCELLigence system is an innovative technology that allows continuous and quantitative monitoring of cells $(11,21)$. The application of microelectrodes in RTCA plates for the measurement of electronic impedance enables observation of the viability of tested cells at every point of time during an experiment. The data are collected throughout the experiment (1). Additionally, there is no need to terminate the experiment and label cells in order to determine $\mathrm{IC}_{50}$ values, which is the case for different end-point assays (22). Furthermore, due to the use of sensor arrays 
in the plates, the RTCA system detects cell changes with higher sensitivity than other cytotoxicity assays $(23,24)$ and gives more information than the single-value end-points obtained in classical tests $(9,25)$. Hence, the xCELLigence system provides an advantage in cytotoxicity research, especially for screening potential anticancer drugs and monitoring of the effects of compounds on cells over hours or even days.

However, despite the many advantages of the RTCA system, its main limitation is that the plates are recommended by the manufacturer to be used only once. As a consequence, the cost of repeated experiments is significantly increased.

We showed that the results obtained with reused E-plates are comparable to those from new plates when analyzing the effects of vinblastine sulfate treatment on HeLa and MCF-7 cells. Furthermore, our experiments using collagen-coated plates showed that the use of this substrate did not influence results from new and used plates.

Coating well surfaces with different substrates is mostly done for RTCA experiments involving adherent cell lines. Fibronectin, collagen, or gelatin are used to improve the adhesion, spreading, and growth of cells, especially those with a weaker ability to attach onto the cell culture well bottoms (26). However, Martinez-Serra et al. have also adapted this methodology of pre-coating the cell culture surface with different substrates for use of the xCELLigence system with leukemia/lymphoma suspension cells (27).

We have demonstrated that E-plates containing wells with sensor-electrodes, which have wide application in many research areas, can be regenerated and reused several times. The regeneration of plates reduces
A
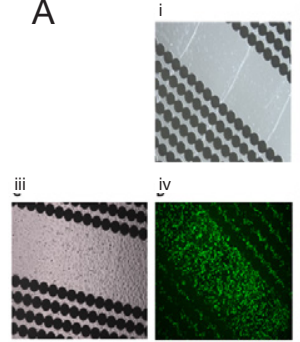

vii

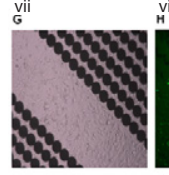



viii

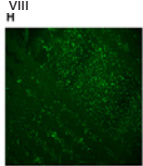

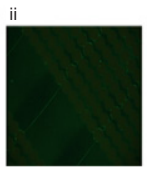
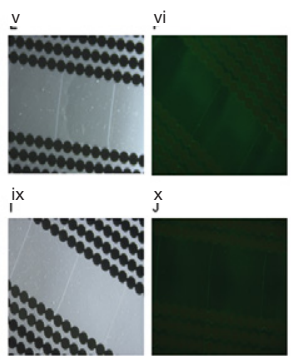

the cost of RTCA experiments and, more importantly, did not affect data reproducibility in our experiments. It has to be stressed that the complete removal of the cytotoxic agent after each use is mandatory, although for some compounds this might not be possible. Despite this limitation, our results provide an alternate solution when the cost of new plates is prohibitive (28). Finally, our experiments assayed the effects of the wellknown cytostatic drug vinblastine (29), but the protocol can be applied to other cytotoxicity studies.

\section{Author contributions}

J.S.H., A.A., and R.B. conceived and designed the experiments. J.S.H. and A.A. performed the experiments. J.S.H., A.A., and R.B. analyzed the data. J.S.H. and A.A. wrote the paper. J.R.O. supervised the study and corrected the manuscript.

\section{Acknowledgments}

This research was supported by the Polish Ministry of Science and Higher Education under grant Nr N N405 669140.

\section{Competing interests}

The authors declare no competing interests.

\section{References}

1. Kustermann, S., F. Boess, A. Buness, M. Schmitz, M. Watzele, T. Weiser, T. Singer, L. Suter, and A. Roth. 2013. A label-free, impedancebased real time assay to identify drug-induced toxicities and differentiate cytostatic from cytotoxic effects. Toxicol. In Vitro 27:1589-1595.

2. Vistejnova, L., J. Dvorakova, M. Hasova, T. Muthny, V. Velebny, K. Soucek, and L. Kubala. 2009. The comparison of impedance-
B
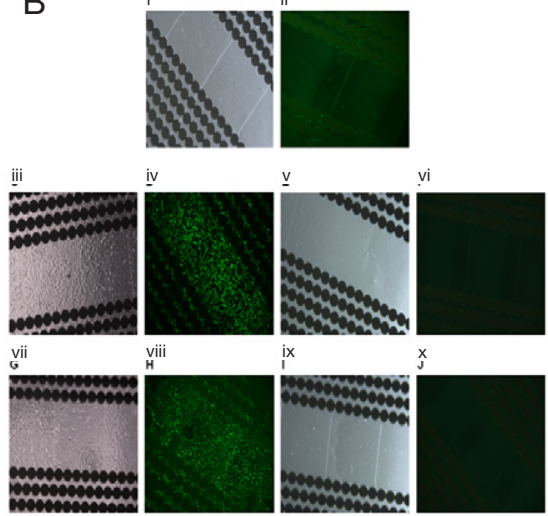

Figure 3. Lectin staining of E-plate surfaces. Plates were either uncoated $(A)$ or coated with collagen (B). Plates were photographed before (i,iii,v,vii, ix) and after (ii, iv,vi,viii, $x$ ) staining with WGA Alexa Fluor conjugate. Images iii, iv, vii, and viii show HeLa cells adhered to the plates; images $v, v i$, ix, and $x$ show plate surfaces after trypsinization. The surfaces of plates (iii- $x$ ) without $(A)$ and with collagen coating $(B)$ were compared with those of the new plates $(i, i i)$.

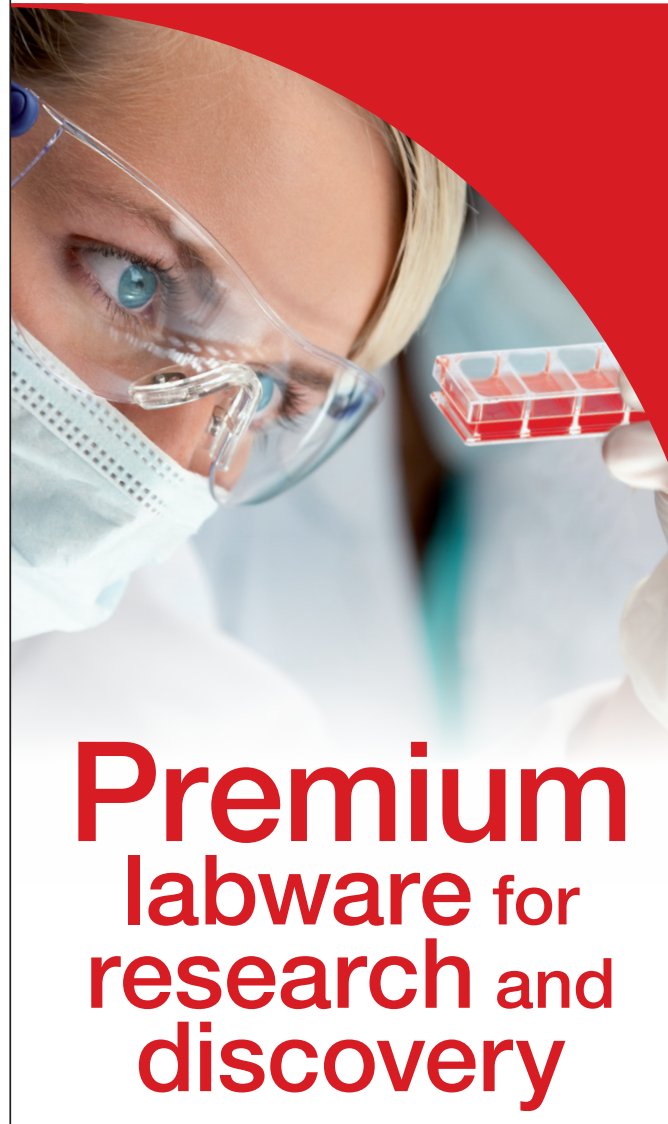

\section{lumox ${ }^{\circledR}$ Technology}

- Very low autofluorescence

- High transparency

- Gas-permeable film base
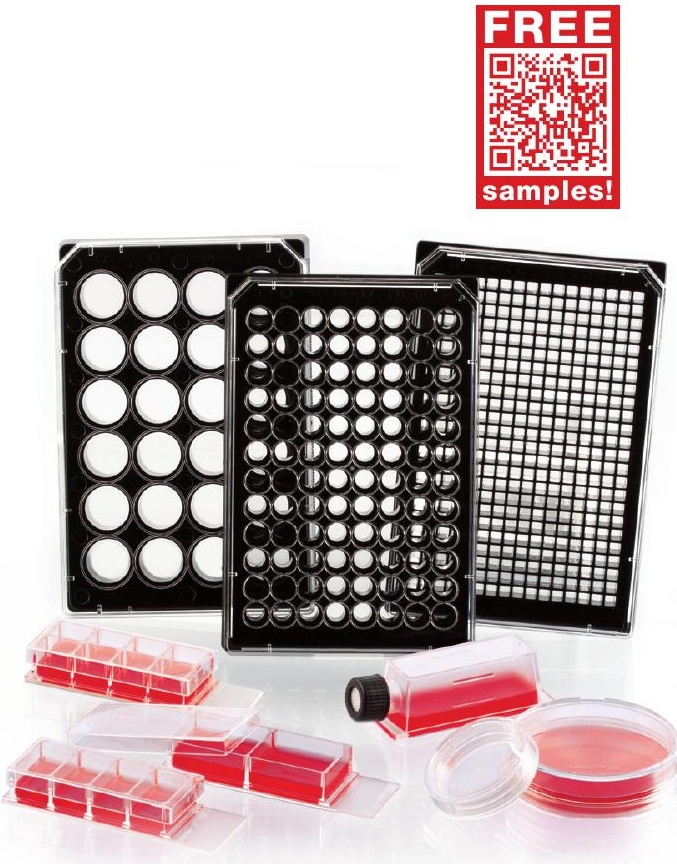
based method of cell proliferation monitoring with commonly used metabolic-based techniques. Neuroendocrinol. Lett. 30:121-127.

3. Dowling, C.M., C. Herranz Ors, and P.A. Kiely. 2014. Using real-time impedance-based assays to monitor the effects of fibroblast-derived media on the adhesion, proliferation, migration and invasion of colon cancer cells. Biosci. Rep. 34:e00126.

4. Hopper, N., J. Wardale, D. Howard, R. Brooks, N. Rushton, and F. Henson. 2015. Peripheral blood derived mononuclear cells enhance the migration and chondrogenic differentiation of multipotent mesenchymal stromal cells. Stem Cells Int. 2015:323454

5. Scrace, S., E. O'Neill, E.M. Hammond, and I.M. Pires. 2013. Use of the xCELLigence system for real-time analysis of changes in cellular motility and adhesion in physiological conditions. Methods Mol. Biol. 1046:295-306.

6. Arias, L.R., C.A. Perry, and L. Yang. 2010. Real-time electrical impedance detection of cellular activities of oral cancer cells. Biosens. Bioelectron. 25:2225-2231.

7. Ramis, G., L. Martinez-Alarcon, J.J. Quereda, L. Mendonca, M.J. Majado, and K. GomezCoelho. 2013. Optimization of cytotoxicity assay by real-time, impedance-based cell analysis. Biomed. Microdevices 15:985-995.

8. Thakur, M., K. Mergel, A. Weng, S. Frech, R. Gilabert-Oriol, D. Bachran, M.F. Melzig, and H. Fuchs. 2012. Real time monitoring of the cell viability during treatment with tumor-targeted toxins and saponins using impedance measurement. Biosens. Bioelectron. 35:503-506.

9. Urcan, E., U. Haertel, M. Styllou, R. Hickel, H. Scherthan, and F.X. Reichl. 2010. Real-time $x$ CELLigence impedance analysis of the cytotox- icity of dental composite components on human gingival fibroblasts. Dent. Mater. 26:51-58.

10. Leme, D.M., T. Grummt, R. Heinze, A. Sehr, M Skerswetat, M.R. de Marchi, M.C. Machado, D.P. de Oliveira, and M.A. Marin-Morales. 2011. Cytotoxicity of water-soluble fraction from biodiesel and its diesel blends to human cell lines. Ecotoxicol. Environ. Saf. 74:2148-2155.

11. Keogh, R.J. 2010. New technology for investigating trophoblast function. Placenta 31:347-350.

12. Braicu, C., C.D. Gherman, A. Irimie, and I. Berindan-Neagoe. 2013. Epigallocatechin3-Gallate (EGCG) inhibits cell proliferation and migratory behaviour of triple negative breast cancer cells. J. Nanosci. Nanotechnol. 13:632-637.

13. Daker, M., M. Ahmad, and A.S. Khoo. 2012 Quercetin-induced inhibition and synergistic activity with cisplatin - a chemotherapeutic strategy for nasopharyngeal carcinoma cells. Cancer Cell Int. 12:34.

14. Stefanowicz-Hajduk, J., R. Bartoszewski, S Bartoszewska, K. Kochan, A. Adamska, I. Kosinski, and J.R. Ochocka. 2015. Pennogenyl saponins from Paris quadrifolia L. induce extrinsic and intrinsic pathway of apoptosis in human cervical cancer HeLa cells. PLoS ONE 10:e0135993.

15. Wei, P.L., C.C. Tu, C.H. Chen, Y.S. Ho, C.T. Wu H.Y. Su, W.Y. Chen, J.J. Liu, and Y.J. Chang. 2013. Shikonin suppresses the migratory ability of hepatocellular carcinoma cells. J. Agric. Food Chem. 61:8191-8197.

16. Wu, J., J. Xu, E.A. Eksioglu, X. Chen, J. Zhou, N. Fortenbery, S. Wei, and J. Dong. 2013. Icariside Il induces apoptosis of melanoma cells through the downregulation of survival pathways. Nutr. Cancer 65:110-117.
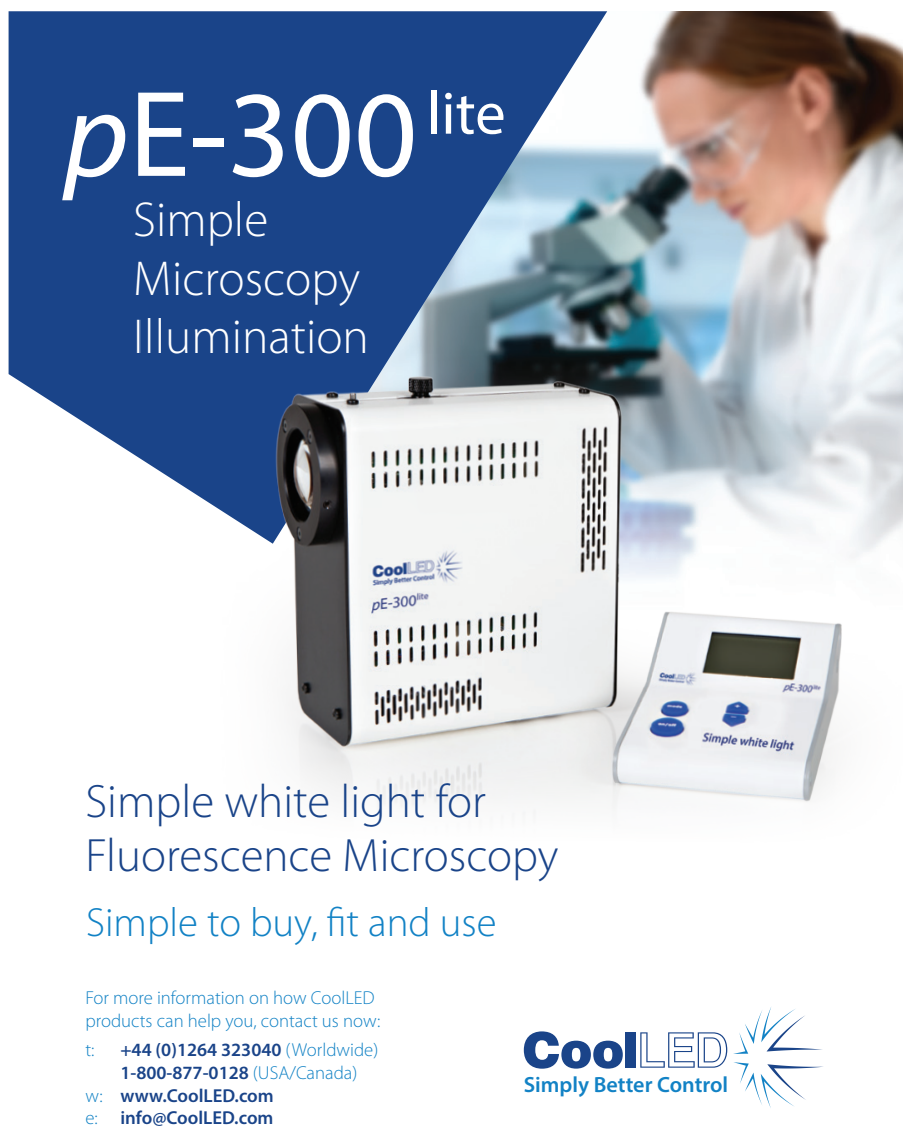

17. Silbereisen, A., L. Tritten, and J. Keiser. 2011. Exploration of novel in vitro assays to study drugs against Trichuris spp. J. Microbiol. Methods 87:169175.

18. Slanina, H., A. König, H. Claus, M. Frosch, and A. Schubert-Unkmeir. 2011. Real-time impedance analysis of host cell response to meningococcal infection. J. Microbiol. Methods 84:101-108.

19. Witkowski, P.T., L. Schuenadel, J. Wiethaus, D.R. Bourquain, A. Kurth, and A. Nitsche. 2010. Cellular impedance measurement as a new tool for poxvirus titration, antibody neutralization testing and evaluation of antiviral substances. Biochem. Biophys. Res. Commun. 401:37-41.

20. Bartoszewski, R., A. Hering, M. Marszałł, J. Stefanowicz-Hajduk, S. Bartoszewska, N. Kapoor, K. Kochan, and J.R. Ochocka. 2014 Mangiferin has an additive effect on the apoptotic properties of hesperidin in Cyclopia sp. tea extracts. PLOS ONE 9:e92128.

21. Ke, N., X. Wang, X. Xu, and Y.A. Abassi. 2011. The $x$ CELLigence system for real-time and labelfree monitoring of cell viability. Methods Mol. Biol. 740:33-43.

22. Limame, R., A. Wouters, B. Pauwels, E. Fransen, M. Peeters, F. Lardon, O. De Wever, and P. Pauwels. 2012. Comparative analysis of dynamic cell viability, migration and invasion assessments by novel real-time technology and classic endpoint assays. PLoS ONE 7:e46536.

23. Caltová, K. and M. Cervinka. 2012. Antiproliferative effects of selected chemotherapeutics in human ovarian cancer cell line A2780. Acta Medica (Hradec Kralove). 55:116-124.

24. Erskine, C.L., A.M. Henle, and K.L. Knutson. 2012. Determining optimal cytotoxic activity of human Her2neu specific CD8 T cells by comparing the $\mathrm{Cr} 51$ release assay to the $\mathrm{XCELLigence} \mathrm{System.}$ J. Vis. Exp. 8:e3683.

25. Malkoç, S., F. Öztürk, B. Çörekçi, B.S. Bozkurt, and S.S. Hakki. 2012. Real-time cell analysis of the cytotoxicity of orthodontic mini-implants on human gingival fibroblasts and mouse osteoblasts. Am. J. Orthod. Dentofacial Orthop. 141:419-426.

26. MacDonald, C., C.P. Unsworth, and E.S. Graham. 2014. Enrichment of differentiated hNT neurons and subsequent analysis using flowcytometry and xCELLigence sensing. J. Neurosci. Methods 227:47-56

27. Martinez-Serra, J., A. Gutierrez, S. MunozCapó, M. Navarro-Palou, T. Ros, J.C. Amat, B. Lopez, T.F. Marcus, et al. 2014. xCELLigence system for real-time label-free monitoring of growth and viability of cell lines from hematological malignancies. Onco Targets Ther. 7:985-994.

28. Kho, D., C. MacDonald, R. Johnson, C.P. Unsworth, S.J. O'Carroll, E. du Mez, C.E. Angel, and E.S. Graham. 2015. Application of XCELLigence RTCA biosensor technology for revealing the profile and window of drug responsiveness in real time. Biosensors (Basel). 5:199-222.

29. Moudi, M., R. Go, C.Y. Yien, and M. Nazre. 2013. Vinca alkaloids. Int J Prev Med. 4:1231-1235.

Received 08 January 2016; accepted 16 June 2016.

Address correspondence to Justyna Stefanowicz-Hajduk, Department of Biology and Pharmaceutical Botany, Medical University of Gdansk, Hallera 107, 80-416, Gdansk, Poland. E-mail: justynastef@gumed.edu.pl

To purchase reprints of this article, contact: biotechniques@fosterprinting.com 